\section{Rhino protection in Africa}

SIR-The Commentary by LeaderWilliams and Albon (Nature 336, 533; 1988), on the allocation of resources for conservation of African big game, leads me to enquire whether any consideration has been given to dehorning of wild rhinoceroses.

For the past 30 years a dehorned male rhinoceros has lived in the National Zoological Park in Washington, DC. Dehorned animals may be handicapped in ritual sparring, and the attractiveness of rhinos for tourists might be marginally reduced (although countered by raised awareness of the poaching problem), but elimination of the rationale for rhino slaughter seems to be a simple and inexpensive measure that might save the species.

JOHN BuCK

4505 Saul Road, Kensington, Maryland 20895, USA

SIR-The Commentary by LeaderWilliams and Albon makes disheartening reading. Sadly, I believe that the prospects may be even gloomier than they suggest.

The authors claim a linear relationship between money spent per square kilometre of habitat and the health of the populations of black rhinos and elephants in Africa. But there is an alternative interpretation of the data presented in their Fig. 4, revolving about the point representing Kenya. My explanation assumes that the Kenyan datum is reliable, an assumption that rests on the fact that the Kenyan national park system is one of the best-run in Africa.

The relationship portrayed is probably not one of linear increase, but is instead 'flat' through levels of spending represented by Kenya (say, less than $\$ 200$ per $\mathrm{km}^{2}$ ) and only begins to show returns when spending levels increase well into the $\$ 200-300$ per $\mathrm{km}^{2}$ range. This is to say that considerable sums must be spent before returns can be expected, and at lower levels there is no reason to expect even minor success. The authors' citation of the relationship between spending and elephant populations (in the caption to Fig. 4) provides a substantially smaller $r^{2}$ value, indicating even less clarity in that relationship.

I see no conflict between this alternative explanation and the data represented in Fig. 3, which show a linear relationship between effort - as $\log _{\mathrm{e}}$ (total patrol days/ size of area/frequency of rhino sightings in 1980) - and the instantaneous rate of change in black rhino populations in the Luangwa Valley. Restricting analysis to a single area offers an important control in a continent as politically and socially diverse as Africa. The continental relationship may likewise be linear, but the data do not yet support that interpretation to the exclusion of others.

The expectation of disappointment at lower spending levels would take some of the bite out of the frustration, and perhaps fuel the argument for greater expenditure on conservation. We are all in for a long haul, and it is high time that both poor and especially rich nations realized that the fight to save African wildlife will continue to be difficult, frustrating and costly.

RANDALL BREITWISCH

Department of Biology,

University of Dayton,

300 College Park,

Dayton, Ohio 45469, USA

LEADER-WilliamS REPLIES-The dehorning of rhinos has been discussed by conservationists since the $1950 \mathrm{~s}$, but has not yet been attempted as a means of preventing poaching. This would require considerable resources, because wild rhinos first have to be caught. The Zimbabwe capture unit, for example, is working to capacity in the Zambezi Valley to remove black rhinos to safer parts of the country and catches around 75 animals per year. Nor has it been established whether dehorning could be permanent, for rhinos which lose a whole horn regrow a new one within 3 years.

Dehorning may also be counterproductive. In the short term, a poacher sighting a rhino in thick bush could shoot before finding his quarry is hornless. In the long term, mother rhinos may be less able to protect their calves from predators, such as lions or hyaenas, thereby reducing recruitment. But given the desperate plight of black rhinos, it may now be appropriate to develop a permanent method of dehorning and at least to monitor the survival of one experimental group of dehorned and control rhinos.

On the basis of one outlying point, Breitwisch argues that the relationship between spending and success at protecting

\section{A new planet around SN1987A}

SIR-The discovery in SN1987A of a 0.508 -ms pulsar, with a sinusoidal $3 \times$ $10^{-3} \mathrm{~Hz}$ (peak-to-peak) modulation of 8hour period, has been reported'. The origin of this modulation is intriguing.

A real modulation in the rotation period of the pulsar by pulsations with an 8 -hour period can be excluded. The modes of pulsation of degenerate white dwarfs are relatively well understood ${ }^{2}$. The upper limit to the period of the longperiod gravity modes is calculated to be around 1,000 seconds for white dwarfs, in agreement with the observations. For a fixed mass the maximum period is directly proportional to the radius of the star. A neutron star has about the same mass as a white dwarf but is three orders of magni- rhinos in different countries may suggest a threshold. In fact, a semilog plot does not provide any better fit to the data for rhinos $\left(r^{2}=0.64\right)$ than the simple regression in our Fig. 4. Furthermore, the regression for elephants (not displayed in our article) had outlying points on both lower and upper sides of the line, as expected in such a politically and socially diverse continent as Africa.

More importantly, there is little disagreement in terms of policy. If resources are scarce, we suggested that they must be concentrated at appropriate levels within small areas to achieve given objectives, in this case to prevent a decline of rhinos. The donation to Zambia, which resulted in the linear relationships for Luangwa Valley in our Fig. 3, achieved little because it was insufficiently concentrated and rhinos declined in all areas at rates far in excess of recruitment. Hence, we did not argue for 'dilute' spending because funds used in this way are, ultimately, wasted. Whether the appropriate sum per unit area is derived from an intercept or from Breitwisch's proposed threshold appears to be immaterial.

We also noted that governments in developing countries and charitable conservation agencies do not have sufficient funds to maintain large conservation areas. It may be less disheartening in future if bilateral aid organizations become increasingly involved in conservation for development. At present, only a small fraction of their annual budgets of around $\$ 40,000$ million are spent on conservation. Even a further 5-10 per cent of their budgets, harnessed into well-directed schemes requested by host governments, would greatly help to stem the erosion of the natural resource base of developing countries.

Large Animal Research Group

N. LEADER-WILLIAMS

Department of Zoology,

34A Storey's Way,

Cambridge CB3 ODT, UK tude smaller, so the maximum pulsation period will be around 1 second.

A natural explanation for the modulation is that it is due to a body orbiting the neutron star. The semi-major axis of the orbit, around a neutron star of one solar mass $\left(M_{\odot}\right)$, of an object with an 8-hour period is 2 solar radii. This implies a mean orbital velocity of such an object of about $300 \mathrm{~km} \mathrm{~s}^{-1}$. The velocity of the pulsar of $0.23 \mathrm{~km} \mathrm{~s}^{-1}$, deduced from the Doppler shift, is only a lower limit to the true velocity, because the inclination is unknown. This gives a lower limit to the mass of the orbiting planet of $7.7 \times 10^{-4} M_{\odot}$; the most likely mass is about twice this (that is, about the mass of Jupiter).

The planet cannot have existed before 